\title{
Improved Use of Inputs to Primordial Nucleosynthesis
}

\author{
Kenneth M. Nollett ${ }^{1,2}$ and Scott Burles ${ }^{2}$ \\ ${ }^{1}$ Department of Physics and ${ }^{2}$ Department of Astronomy and \\ Astrophysics, The University of Chicago, 5640 S. Ellis Ave., Chicago, \\ IL 60637, USA
}

\begin{abstract}
We present a new method to incorporate laboratory nuclear data directly into the standard big-bang nucleosynthesis calculation. Using the quoted uncertainties on the data in a Monte Carlo procedure, we estimate likelihood distributions of the predicted abundances. Our results indicate significantly narrower confidence limits for the predictions than those presently in use. This technique provides error estimates that become smaller as more input data are added, and it allows new nuclear data to be incorporated easily as they become available.
\end{abstract}

Calculations of the primordial abundances in the standard big bang nucleosynthesis (BBN) framework require as inputs the thermal reaction rates for twelve key nuclear reactions. The results of the standard BBN calculation are limited in accuracy almost exclusively by the accuracy of these rates. The quoted uncertainties on the observed $\mathrm{D}$ and ${ }^{7} \mathrm{Li}$ abundances are now smaller than the uncertainties quoted on most standard BBN calculations.

Our new method is intended to make the most direct possible use of the nuclear data and their published uncertainties, to be explicitly less conservative and less subjective than the previous evaluations (while still being reasonable), and to take correlated uncertainties into account. Perhaps most importantly, we wanted our method to reduce the size of the formal abundance uncertainties as newer, more accurate laboratory data are added. It is important to note that the current standard evaluation of the uncertainties in ? set many of its error estimates by requiring that all published data lie within the formal two-sigma errors on the cross section. While this is fine for a one-time conservative error estimate, it can not be re-applied to a growing database in a way that reduces error estimates as data are added, unless some old data are discarded.

We examined the entire experimental literature on the key reactions from approximately 1945 onward, to ensure that we were using the right data, and that we were not mis-using them. We then used the data to obtain abundance predictions by a Monte Carlo process. Each abundance calculation contained the following steps:

1) For each measured cross section (data point), a random number was drawn from a Gaussian distribution whose mean was the measured cross section and whose standard deviation was the (independent) uncertainty for that point. For each set of points sharing a common normalization, a normalization was drawn from a Gaussian distribution with standard deviation given by the normalization error for that set of points. 
2) An integrable function was generated from these data by a weighted least-squares fit of the $S$-factor to a piecewise polynomial. Thermally averaged reaction rates were then generated.

3) The thermal reaction rates were used as inputs to a standard BBN code, which evolved the reaction network to produce primordial abundances of ${ }^{4} \mathrm{He}$, $\mathrm{D},{ }^{3} \mathrm{He}$, and ${ }^{7} \mathrm{Li}$.

After 25,000 repetitions of this procedure, we examined the distribution of output abundances and extracted the $90 \%$ confidence limits corresponding to the input cross section distributions. For more thorough descriptions of our procedure, see ? and ?.

The median abundances generated by our procedure differ little from the previous standard, although there are cases where new measurements tend to produce some deviation $\left(d(p, \gamma){ }^{3} \mathrm{He},{ }^{3} \mathrm{He}(n, p){ }^{3} \mathrm{H}\right)$, as well as cases where existing data sets receive different weighting than in the previous evaluation (the $d+d$ reactions, ${ }^{3} \mathrm{He}(\alpha, \gamma){ }^{7} \mathrm{Be}$, and $\left.{ }^{3} \mathrm{He}(n, p){ }^{3} \mathrm{H}\right)$. These changes tend to cancel each other in the cases of $\mathrm{D}$ and $\mathrm{Li}$ abundances, but not in the case of ${ }^{3} \mathrm{He}$. More notably, we arrive at narrower limits on the $\mathrm{D},{ }^{3} \mathrm{He}$ and ${ }^{7} \mathrm{Li}$ predictions, with $2 \sigma$ errors estimated to be smaller by up to a factor of three than in the previous, conservative, estimate.

The greatest strength of our method is its close coupling to the nuclear data. It uses only the most relevant data for a given temperature, and it allows new data to be incorporated easily into the calculation. To identify which reactions are contributing to the uncertainties and to the differences from the previous standard rates, we ran the Monte Carlo code several more times. For each of these runs, we set ten of the key reaction rates to their values in ? while we treated only one reaction with our Monte Carlo technique.

Combining the reaction-by-reaction analysis with the study of the reaction rate sensitivitites in ?, we conclude that the places where very precise cross sections would be most useful are as follows: For deuterium, $p(n, \gamma) d$ at 20-300 $\mathrm{keV}, d(d, n)^{3} \mathrm{He}$ above $100 \mathrm{keV}, d(d, p)^{3} \mathrm{H}$ above $100 \mathrm{keV}$, and $d(p, \gamma)^{3} \mathrm{He}$ everywhere, while relative importance varies with baryon density. For lithium at $\Omega_{B} h^{2}=0.019$, the most important reactions, in order, are $p(n, \gamma) d$ at $20-150$ $\mathrm{keV},{ }^{3} \mathrm{He}(\alpha, \gamma){ }^{7} \mathrm{Be}$ at $150-380 \mathrm{keV}$ (and overall normalization), $d(p, \gamma)^{3} \mathrm{He}$ everywhere, and $d(d, n)^{3} \mathrm{He}$ above $100 \mathrm{keV}$. For lithium at $\Omega_{B} h^{2}=0.009$, the most important contributions are from ${ }^{3} \mathrm{H}(\alpha, \gamma){ }^{7} \mathrm{Li}$ at $40-300 \mathrm{keV}$ and ${ }^{7} \mathrm{Li}(p, \alpha)^{4} \mathrm{He}$ at $40-300 \mathrm{keV}$.

\section{References}

Burles, S., Nollett, K. M., Truran, J. W., \& Turner, M. S. 1999, Phys.Rev.Lett, 82,4176

Nollett, K. M. \& Burles, S. 1999, submitted to Phys.Rev.D

Smith, M. S., Kawano, L. H., \& Malaney, R. A. 1993, ApJS, 85, 219 


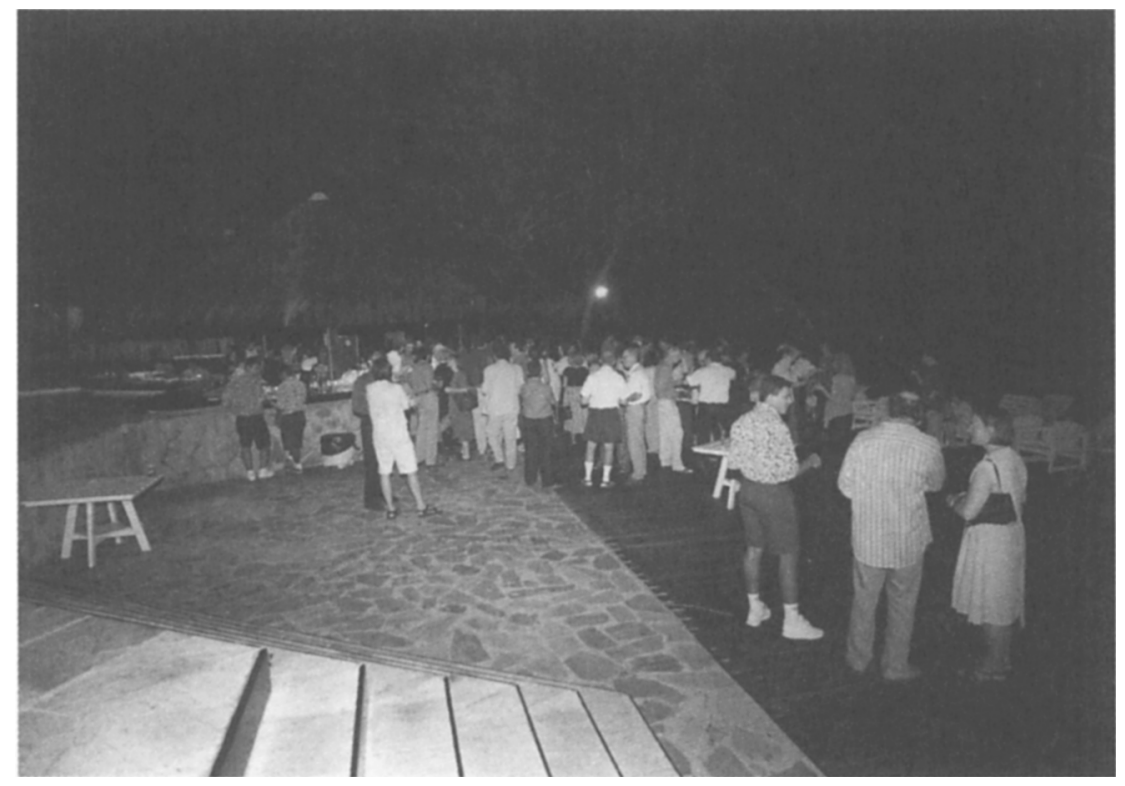

The welcome reception, in a pleasant Springtime evening, typical of Natal. 